\title{
Evidence of a new intermediate compound of the chitin biogenesis found in the marine-derived Penicilliumroqueforti fungus
}

\author{
Francisco Javier Toledo Marante ${ }^{1}$, Roberto Mioso ${ }^{2 *}$, Irma Herrera Bravo de Laguna ${ }^{3}$, Néstor Vicente Torres ${ }^{4}$ \\ From 5th Congress of the Brazilian Biotechnology Society (SBBIOTEC) \\ Florianópolis, Brazil. 10-14 November 2013
}

Chitin derivatives, chitosan and substituted chito-oligosaccharides from fungi present a wide spectrum of applications and they have been studied in many fields such as medicine, cosmetics, agriculture, aquaculture, and food as dietary supplements [1]. Chitin is a copolymer with $N$-acetyl-D-glucosamine units linked with $\beta$ (1-4)-glycosidic bonds which provide rigidity to the cell wall in chitinous fungi. There is evidence that the chitin synthesis is catalyzed by the chitin synthase (CS; EC 2.4.1.16), an enzyme that transfers $\beta$-1,4-linked anhydro2-acetamido-2-deoxy-D-glucose (GlcNAc) from uridine diphosphate $\mathrm{N}$-acetylglucosamine (UDP-GlcNAc) to the nonreducing end of growing chitin chains [2]. However, chitin is synthesized by the regulation of distinct isoenzymes whose number ranges in some hyphomycetes [3]. Nevertheless, there is relatively little information on the genes responsible for chitin biosynthesis in filamentous fungi, analyses of DNA fragments from taxonomically diverse fungal species have shown that most fungi have three to six chitin synthase genes [4]. Therefore, this diversity of chitin synthases makes difficult to find a unique model of regulation of the chitin pathway. The chemical screening of the biomass of a new marinederived strain of Penicilliumroqueforti, produced by liquid-state fermentation, led to the identification of several volatile and non-volatile compounds [5]. As a result of this previous study, we have isolated and characterized a new molecule. The chemical structure of the 2deoxy-2-phosphamino- $\alpha$-D-glucopyranose isolated was elucidated on the basis of 1D and 2D NMR studies as well as other instrumental techniques. In consequence of this discovery, it has been proposed a biogenetic route aiming to explain its formation as an intermediary component of the chitin biosynthesis.

\section{Authors' details}

'Departamento de Química, Universidad de Las Palmas de Gran Canaria, Gran Canaria, 35017, Spain. ${ }^{2}$ Centro de Biotecnologia, Universidade Federal da Paraíba, João Pessoa, Paraíba, 58051-970, Brazil. ${ }^{3}$ Departamento de Biología, Universidad de Las Palmas de Gran Canaria, Gran Canaria, 35017 Spain. ${ }^{4} G$ rupo de Tecnología Bioquímica, Departamento de Bioquímica y Biología Molecular, Instituto de Tecnología Biomédica, Universidad de La Laguna, San Cristóbal de La Laguna, Tenerife, 38206, Spain.

Published: 1 October 2014

References

1. Jayakumar R, Menon D, Manzoor K, Nair SV, Tamura H: Biomedical applications of chitin and chitosan based nanomaterials - A short review. Carbohyd Polym 2010, 82(2):227-232.

2. Bulik DA, Olczak M, Lucero HA, Osmond BC, Robbins PW, Specht CA: Chitin synthesis in Saccharomyces cerevisiae in response to supplementation of growth medium with glucosamine and cell wall stress. Eukaryot Cell 2003, 29(5):886-900.

3. Hartl L, Zach S, Seidl-Seiboth V: Fungal chitinases: diversity, mechanistic properties and biotechnological potential. Appl Microbiol Biot 2012, 93(2):533-43.

4. Gandía M, Harries E, Marcos JF: Identification and characterization of chitin synthase genes in the postharvest citrus fruit pathogen Penicillium digitatum. Fungal Biol 2012, 116(6):654-664.

5. Mioso R, Toledo Marante FJ, Bravo de Laguna IH, González JEG, Rodríguez JJS: Biomolecules produced in liquid-state fermentation by a marine-derived fungus, Penicillium roqueforti. Química Nova, accepted.

doi:10.1186/1753-6561-8-S4-P212

Cite this article as: Marante et al:: Evidence of a new intermediate compound of the chitin biogenesis found in the marine-derived Penicilliumroqueforti fungus. BMC Proceedings 2014 8(Suppl 4):P212. 